\title{
Aplicação da metodologia GDE/UnB para avaliação do grau de deterioração estrutural de edificação - Estudo de caso em Salvador/BA
}

\author{
L. N. CAIRES ${ }^{1}$, V. A. COELHO ${ }^{2 *}$, A. R. A. OMORE ${ }^{3}$, M. A. MACHADO ${ }^{3}$, \\ P. V. G. FREITAS ${ }^{4}$, F. G. S. SILVA ${ }^{5}$ \\ *Autor de Contato: mrvoelho@gmail.com

\begin{abstract}
${ }^{1}$ Pós-graduando em Engenharia de Avaliação e Perícia, Universidade Jorge Amado, Salvador, Brasil ${ }^{2}$ PPEC, Escola Politécnica, Universidade Federal da Bahia, Salvador, Brasil

${ }^{3}$ Graduado(a) em Engenharia Civil, Escola Politécnica, Universidade Federal da Bahia, Salvador, Brasil

${ }^{4}$ Área de construção civil, SENAI CIMATEC, Salvador, Brasil

${ }^{5}$ DCE, Escola Politécnica, Universidade Federal da Bahia, Salvador, Brasil
\end{abstract}

\begin{abstract}
RESUMO
O desempenho das edificações está ligado à sua capacidade de resistir às ações agressivas do meio em que estão inseridas, sendo fundamental para a continuidade de seu uso e garantia de sua segurança que sejam efetuados os procedimentos periódicos de manutenção. As metodologias de diagnóstico do estado de degradação das estruturas auxiliam na identificação do grau de dano e da classificação da urgência de realização de intervenções. Neste sentido, o presente trabalho utilizou a metodologia GDE/UnB para avaliar as manifestações patológicas existentes no pavimento garagem de uma edificação próxima ao mar, na cidade de Salvador, BA. Foram identificadas, majoritariamente, a corrosão das armaduras e a fissuração da estrutura, classificada, conforme o método, com o nível crítico de deterioração, situação em que a necessidade de intervenção é imediata.
\end{abstract}

Palavras-chave: Degradação; GDE/UnB; Manutenção; Inspeção. 


\title{
Application of the GDE/UnB methodology to assess the degree of structural deterioration of a building - Case study in Salvador/BA
}

\begin{abstract}
The performance of buildings is connected to their ability to resist the aggressive actions of the environment in which they are inserted. The periodic maintenance procedures of buildings are essential for the continuity of their use and guarantee of safety. Methodologies for diagnosing the state of deterioration of structures help to identify the degree of damage and classify the urgency of interventions. In this sense, the present work used the GDE/UnB methodology to evaluate the pathological manifestations existing in the garage of a building close to the sea in the city of Salvador-BA. Corrosion of the reinforcement and cracking of the structure were mainly identified and the structure were classified with a critical level of deterioration according to the method, a situation in which the need for intervention is immediate.
\end{abstract}

Palavras-chave: Degradation; GDE/UnB; Maintenance; Inspection.

\section{Aplicación de la metodología GDE/UnB para evaluar el grado de deterioro estructural de un edificio - Estudio de caso en Salvador / BA}

\section{RESUMEN}

El desempeño de los edificios está ligado a su capacidad para resistir las acciones agresivas del entorno en el que se insertan, siendo fundamental para la continuidad de su uso y garantía de seguridad que se realicen procedimientos periódicos de mantenimiento. Las metodologías para diagnosticar el estado de deterioro de las estructuras ayudan a identificar el grado de daño y clasificar la urgencia de realizar las intervenciones. En este sentido, el presente trabajo utilizó la metodología GDE / UnB para evaluar las manifestaciones patológicas existentes en el pavimento de garaje de un edificio cercano al mar en la ciudad de Salvador-BA. Se identificaron principalmente la corrosión del refuerzo y la fisuración de la estructura, clasificada según el método con un nivel crítico de deterioro, situación en la que la necesidad de intervención es inmediata.

Palavras-chave: Degradación; GDE/UnB; Mantenimiento; Inspección.

\section{INTRODUÇÃO}

O desempenho das edificações é entendido como sendo a capacidade destas e de seus sistemas constituintes em resistir às ações do meio em que estão inseridas, juntamente às solicitações do uso para as quais foram projetadas (ABNT, 2013). Há uma redução natural do desempenho das construções, ao longo de sua vida útil, em função do desgaste de seus sistemas. Contudo, há situações em que os fenômenos de degradação são amplificados, seja pelo uso inadequado de materiais, das falhas previstas em projeto, da execução ou da ausência de manutenção adequada. Neste cenário, ocorre a degradação prematura das edificações com aumento progressivo dos custos de manutenção e reabilitação de acordo com o grau de evolução das manifestações patológicas, 
além do comprometimento da usabilidade e segurança da estrutura (LIMA et al., 2019; POSSAN, DEMOLINER, 2013).

Assim, cabe destacar a importância das atividades de manutenção, fundamentais para a continuidade das condições de uso das edificações dentro dos níveis desejados de desempenho e de segurança. A ausência de tais práticas, especialmente as de caráter preventivo, prejudicam o bom funcionamento dos sistemas construtivos, acelerando, ainda mais, os danos causados pelos fenômenos deletérios, sendo a adoção destas intervenções uma prática ainda incipiente no cenário nacional (VILLANUEVA, 2015; OLIVEIRA et al., 2018)

O uso de metodologias de diagnóstico do estado de degradação das estruturas atua, fortemente, para a mudança deste cenário, auxiliando, de forma prática e eficaz, a tomada de decisões quanto às práticas de manutenção apropriadas para cada situação, permitindo, ainda, que as manifestações patológicas presentes sejam classificadas quanto à sua gravidade e à urgência de intervenção (LIMA et al., 2019)

A metodologia GDE/UnB propõe a avaliação da estrutura de forma global e localizada por meio de um roteiro de inspeção. Neste, a estrutura é dividida em famílias de elementos estruturais, onde cada elemento é avaliado quanto às possíveis manifestações patológicas existentes e, por sua vez, classificados quanto ao grau de deterioração. As manifestações são ponderadas de acordo com a gravidade que representam para a funcionalidade da família e gravidade dos danos registrados na estrutura avaliada. Os resultados individuais são combinados e fornecem indicadores importantes sobre a deterioração dos elementos, das famílias estruturais e da estrutura como um todo, classificando cada um destes grupos em níveis prioritários para realização de intervenção (FONSECA, 2004).

Proposta inicialmente por Castro (1994), a metodologia GDE/UnB foi aprimorada para diversos usos, sendo adequada para a inspeção de variadas estruturas em concreto armado, desde as edificações convencionais até as obras de arte consideradas especiais (LIMA et al., 2019; GASPARETTO et al., 2021; OLIVEIRA et al., 2018; GALVÃO, SILVA, 2021)

Dado o exposto, o presente estudo de caso tem como objetivo avaliar as manifestações patológicas presentes em uma edificação residencial situada próxima ao mar, fazendo uso da metodologia GDE/UnB para determinar o nível de deterioração da estrutura, bem como estipular o período mínimo recomendado para realização de manutenções.

\section{METODOLOGIA}

\subsection{Objeto de estudo}

A investigação deste trabalho está baseada na análise do pavimento térreo de uma edificação residencial de quatro pavimentos, situada na cidade de Salvador, BA, sendo esta utilizada como edifício garagem. A estrutura possuía aproximadamente 40 anos quando da ocorrência da vistoria, cerca de $380 \mathrm{~m}^{2}$ de área construída, distância de apenas $40 \mathrm{~m}$ até o mar, sistema construtivo em concreto armado com alvenaria de vedação e acabamento de fachada em pintura.

A proximidade com o mar coloca a estrutura na classe de agressividade ambiental IV da NBR 6118 (ABNT, 2014). A Figura 1 apresenta um croqui do pavimento vistoriado, nela pode-se observar que a estrutura dispõe de 44 pilares, 32 vigas e 9 lajes, sendo o revestimento dos pilares constituído por textura sobre pastilhas cerâmicas e as vigas e pilares revestidas apenas com pintura. 


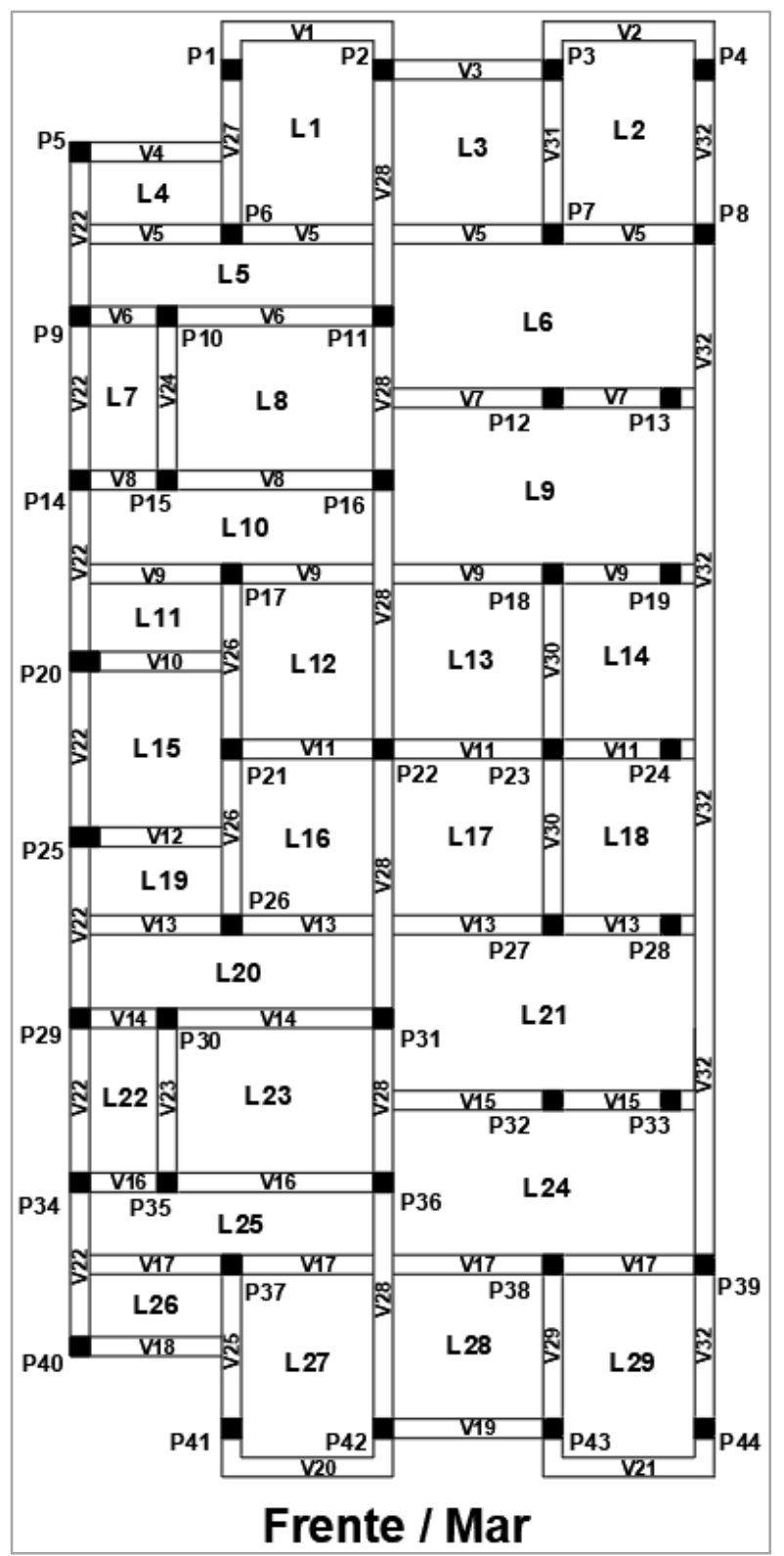

Figura 1 - Croqui da estrutura da garagem

\subsection{Metodologia}

A metodologia consistiu na inspeção visual in loco associada ao método de avaliação GDE/UnB com base nas diretrizes propostas por Fonseca (2007) para verificação dos danos existentes na estrutura. Durante aplicação do método, foi feita inicialmente a divisão e quantificação dos elementos estruturais, separando-os por famílias de elementos de acordo com sua finalidade estrutural, seguida pela inspeção de cada elemento, com base nas orientações de um caderno de inspeção, sendo atribuídos pesos a cada tipo de manifestação patológica de acordo com o elemento em que se encontra e sua gravidade.

Após cálculo do fator de dano, tem-se um indicativo valioso sobre o estado individual de cada elemento e da estrutura como um todo, sendo possível a categorização do nível de deterioração e recomendação da urgência para tomada de ações preventivas e corretivas.

A Figura 2 apresenta o fluxograma de aplicação do método proposto por Fonseca (2007), sendo suas etapas brevemente descritas em sequência. 


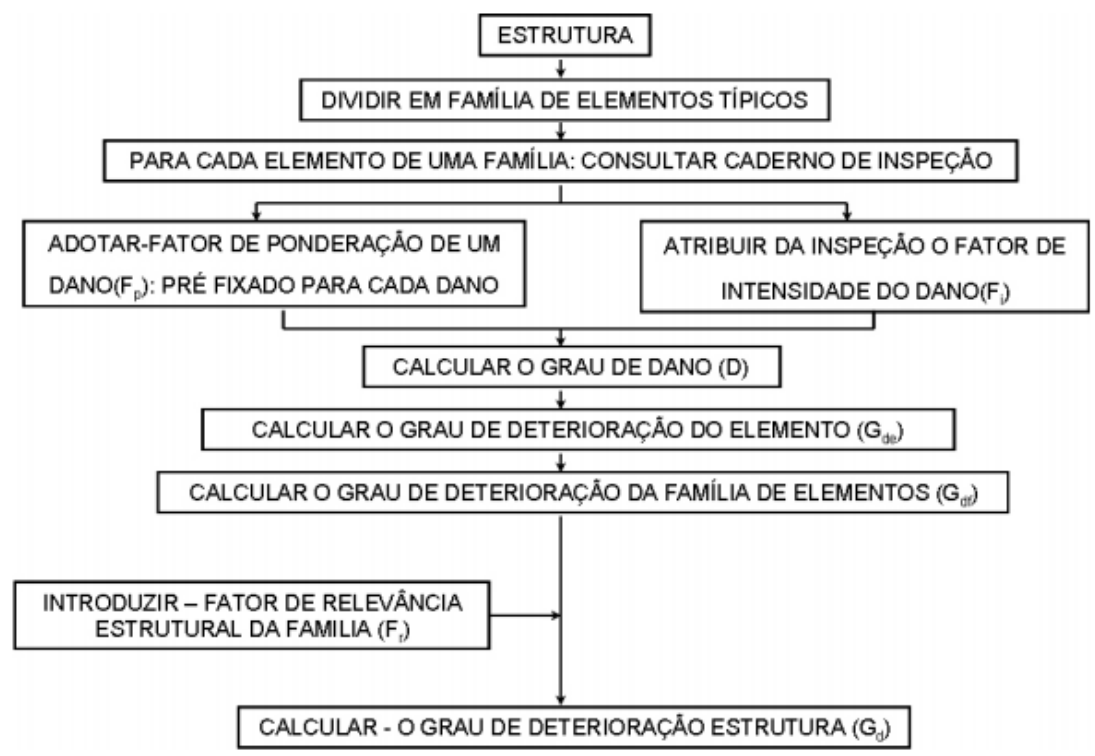

Figura 2 - Fluxograma de aplicação da metodologia GDE/UnB. Adaptado de Fonseca (2007)

O caderno de inspeções do método GDE/UnB fornece os valores de ponderação de dano (Fp) para cada manifestação patológica em relação ao elemento estrutural onde se encontra. $\mathrm{O}$ fator de intensidade (Fi) é determinado pelo avaliador, de acordo com o estado de evolução da manifestação sendo classificada. O caderno de inspeções traz ainda exemplos para auxiliar na determinação do valor de Fi, variando 0 (Inexistente) à 4 (Estado crítico).

Determinados tais fatores, calcula-se o grau de dano associado a cada manifestação por meio da equação (1).

$$
\begin{array}{ll}
D=(0,8 * F i * F p) & \text { para } F i \leq 2 \\
D=[(12 * F i-28) * F p] & \text { para } F i>2
\end{array}
$$

Em seguida, é determinado o grau de deterioração do elemento (Gde) com a expressão (2), onde "m" representa a quantidade de danos identificados no elemento. Tal indicador é importante para a análise da estrutura e pode ser utilizado para determinar a necessidade de intervenção em cada elemento de forma individual.

$$
G d e=D \text { máx } *\left[1+\frac{\sum_{i=1}^{m} D(i)-D \text { máx }}{\sum_{i=1}^{m} D(i)}\right]
$$

A próxima etapa da metodologia determina que seja calculado o grau de deterioração de uma família de elementos (Gdf) com base na expressão (3), agrupando-os por grupo estrutural (pilares, vigas, lajes etc.). Importante observar que só contribuem para esta expressão aqueles elementos com Gde maior ou igual a 15. 


$$
G d f=G d e m a ́ x *\left[\sqrt{\left.1+\frac{\sum_{i=1}^{m} G d e(i)-G d e m a ́ x}{\sum_{i=1}^{m} G d e(i)}\right]}\right]
$$

Por fim, determina-se o grau de deterioração da estrutura (Gd) com base na equação (4). Nesta, Fr representa o fator de relevância estrutural, um coeficiente tabelado no caderno de inspeção que busca ponderar a importância relativa de cada família de elementos no comportamento e bom desempenho da estrutura, variando de 1 (elementos arquitetônicos) a 5 (vigas e pilares principais).

$$
G d=\frac{\sum_{i=1}^{K} F r(i) * G d f(i)}{\sum_{i=1}^{K} F r(i)}
$$

Considera-se que estruturas com valor Gd menores que 15 apresentam baixo nível de deterioração, necessitando apenas de manutenção preventiva. Por outro lado, um Gd maior que 100 caracteriza um estado de degradação crítico, onde medidas emergenciais devem ser adotadas. As classificações do método são apresentadas na Tabela 1.

Tabela 1 - Nível de deterioração da estrutura

\begin{tabular}{|c|c|l|}
\hline Gd & $\begin{array}{c}\text { Nível de } \\
\text { deterioração }\end{array}$ & \multicolumn{1}{c|}{ Ações recomendadas } \\
\hline Menor que 15 & Baixo & Manutenção preventiva \\
\hline $15-50$ & Médio & $\begin{array}{l}\text { Definir prazo para nova inspeção. } \\
\text { Planejar inspeção em longo prazo (máximo 2 anos) }\end{array}$ \\
\hline $50-80$ & Alto & $\begin{array}{l}\text { Definir prazo para inspeção especializada. } \\
\text { Planejar intervenção em médio prazo (máximo 1 ano) }\end{array}$ \\
\hline $80-100$ & Sofrível & $\begin{array}{l}\text { Definir prazo para inspeção especializada rigorosa. } \\
\text { Planejar intervenção em curto prazo (máximo 6 meses) }\end{array}$ \\
\hline Maior que 100 & Crítico & $\begin{array}{l}\text { Inspeção especializada imediata e adoção de medidas } \\
\text { emergenciais. }\end{array}$ \\
\hline
\end{tabular}

Adaptado de Fonseca (2007)

\section{RESULTADOS E DISCUSSÃO}

\subsection{Inspeção visual}

As figuras 3 a 8 ilustram as principais manifestações patológicas encontradas no pavimento vistoriado. Destacam-se grandes fissurações decorrentes da corrosão avançada das armaduras, deformações excessivas de algumas vigas (que foram escoradas) e o desplacamento do concreto. Sendo que foi constatado, durante a vistoria, a existência de cobrimento insuficiente das armaduras para a classe ambiental da estrutura, variando entre 10 e $20 \mathrm{~mm}$. 


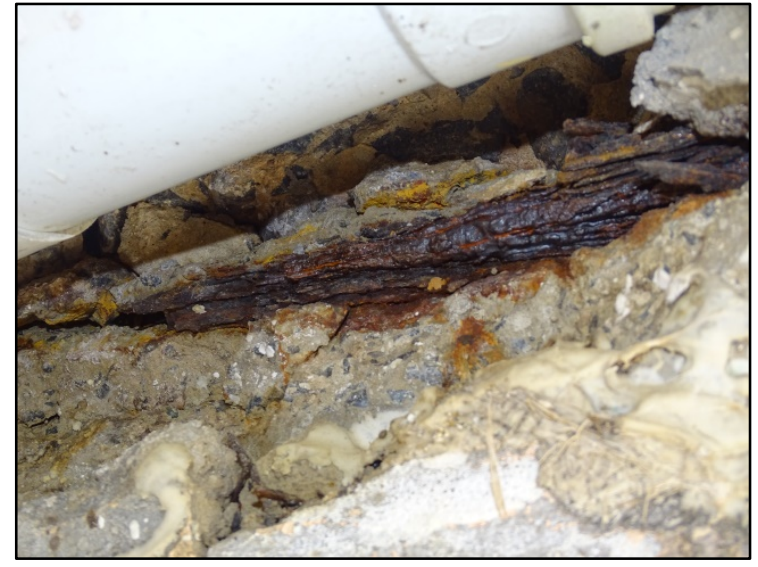

Figura 3 - Corrosão na viga V22

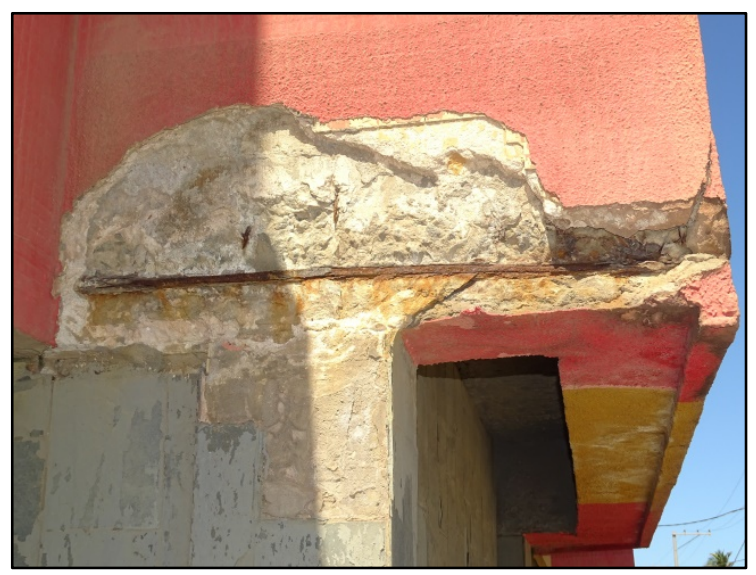

Figura 5 - Desplacamento e corrosão, V25

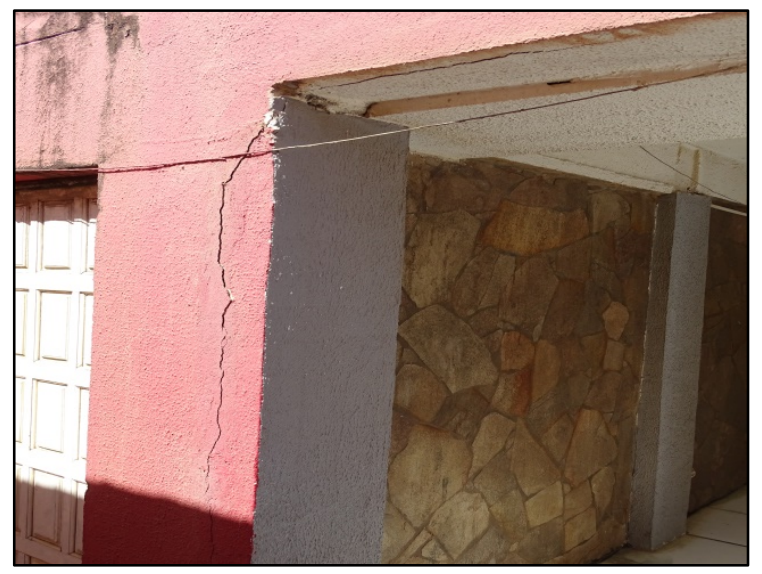

Figura 7 - Fissuração, P14

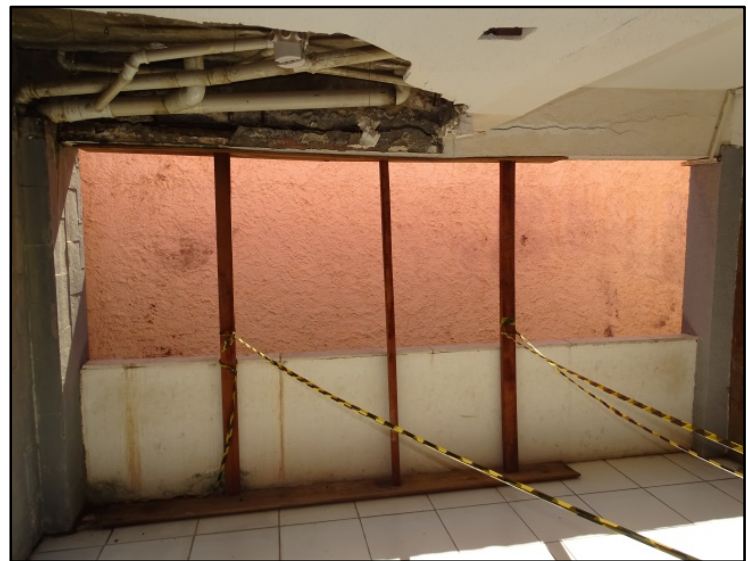

Figura 4 - Desplacamento e fissuras, V22

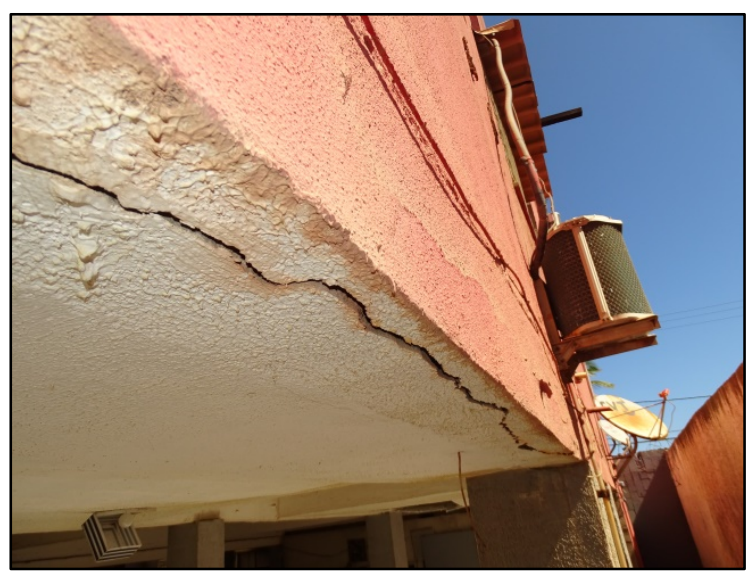

Figura 6 - Fissuração no fundo da viga

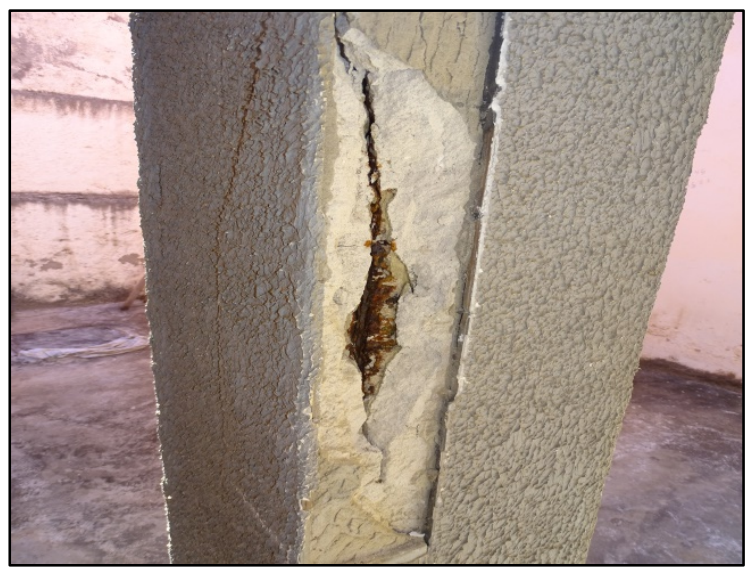

Figura 8 - Desplacamento com corrosão, P1

\subsection{Determinação do grau de deterioração}

Neste trabalho, considerou-se um valor de $\mathrm{Fp}=5$ para as fissuras, em virtude de estarem todas associadas à corrosão das armaduras, uma situação de grande relevância na deterioração das estruturas em ambiente com presença de cloretos, e por não existir valor tabelado para tal cenário no caderno de inspeção. Os valores de Fp para as demais manifestações seguiram a sugestão do caderno de inspeção da metodologia GDE/UnB.

Quanto ao Fi, adotou-se valor 4 (crítico) para as fissuras com grande abertura, onde era possível visualizar a armadura do elemento estrutural com estado corrosivo avançado, e 3 (Grave) para as 
demais. Para as manifestações de carbonatação e ataque por íons cloreto também se definiu Fi $=4$, em concordância com os exemplos apresentados pelo caderno de inspeções tomando como base a situação encontrada de exposição das armaduras a uma maior concentração de gases devido ao desplacamento do concreto e proximidade do mar.

O cálculo do grau de deterioração dos elementos é ilustrado na Tabela 2, que traz os resultados encontrados na viga V22, selecionada por ser o elemento visualmente mais danificado, apresentando o maior Gde. As Tabela 3 e Tabela 4 apresentam as tabelas de cálculo dos indicadores Gdf e Gd para as famílias e estrutura, respectivamente.

Tabela 2 - Avaliação da deterioração da viga V22

\begin{tabular}{|c|c|c|c|c|}
\hline & Nome da Peça & \multicolumn{3}{|c|}{ V22 } \\
\hline \multicolumn{2}{|r|}{ Local } & \multicolumn{3}{|c|}{ Garagem } \\
\hline Item & Patologias & $\mathrm{Fp}$ & $\mathrm{Fi}$ & D \\
\hline 1 & Carbonatação & 3 & 4 & 60 \\
\hline 2 & Cobrimento Deficiente & 3 & 3 & 24 \\
\hline 3 & Contaminação por Cloretos & 4 & 4 & 80 \\
\hline 4 & Corrosão de Armaduras & 5 & 4 & 100 \\
\hline 5 & Desplacamento & 3 & 4 & 60 \\
\hline 6 & Fissuras & 5 & 4 & 100 \\
\hline 7 & Flechas & 5 & 4 & 100 \\
\hline 8 & Umidade & 3 & 3 & 24 \\
\hline \multicolumn{3}{|c|}{ Grau de Deterioração do Elemento (GDE) } & \multicolumn{2}{|c|}{181,75} \\
\hline \multicolumn{3}{|c|}{ Nível de Deterioração do Elemento } & \multicolumn{2}{|c|}{ CRÍTICO } \\
\hline
\end{tabular}

Tabela 3 - Grau de deterioração das famílias

\begin{tabular}{|c|c|c|}
\hline \multicolumn{3}{|c|}{ PILARES } \\
\hline Quantidade de Elementos & \multicolumn{2}{c|}{44} \\
\hline Soma do GDE & \multicolumn{2}{c|}{5680,49} \\
\hline Máximo GDE & 176,42 & P1 e P19 \\
\hline Grau de Deterioração da Família (GDF) & \multicolumn{2}{c|}{$\mathbf{2 4 7 , 5 4}$} \\
\hline
\end{tabular}

\begin{tabular}{|c|c|c|}
\hline \multicolumn{2}{|c|}{ VIGAS } \\
\hline Quantidade de Elementos & \multicolumn{2}{c|}{28} \\
\hline Soma do GDE & \multicolumn{2}{c|}{4107,94} \\
\hline Máximo GDE & 181,75 & V22 \\
\hline Grau de Deterioração da Família (GDF) & \multicolumn{2}{c|}{254,18} \\
\hline
\end{tabular}

\begin{tabular}{|c|c|c|}
\hline \multicolumn{3}{|c|}{ VIGAS SECUNDÁRIAS } \\
\hline Quantidade de Elementos & \multicolumn{2}{c|}{4} \\
\hline Soma do GDE & \multicolumn{2}{c|}{643,32} \\
\hline Máximo GDE & 176,42 & V20 e V21 \\
\hline Grau de Deterioração da Família (GDF) & \multicolumn{2}{c|}{$\mathbf{2 3 1 , 7 5}$} \\
\hline
\end{tabular}

\begin{tabular}{|c|c|c|}
\hline \multicolumn{2}{|c|}{ LAJES } \\
\hline Quantidade de Elementos & \multicolumn{2}{c|}{29} \\
\hline Soma do GDE & \multicolumn{2}{c|}{2907,78} \\
\hline Máximo GDE & 175,25 & L22 \\
\hline Grau de Deterioração da Família (GDF) & \multicolumn{2}{c|}{$\mathbf{2 4 4 , 0 7}$} \\
\hline
\end{tabular}


Tabela 4 - Grau de deterioração da estrutura

\begin{tabular}{|l|c|c|c|}
\hline \multicolumn{4}{|c|}{ AVALIAÇÃO DA ESTRUTURA } \\
\hline \multicolumn{1}{|c|}{ Famílias } & Gdf & Fr & Gdf x Fr \\
\hline Pilares & 247,54 & 5 & 1237,72 \\
\hline Vigas & 254,18 & 5 & 1270,88 \\
\hline Vigas Secundárias & 231,75 & 4 & 927,02 \\
\hline Lajes & 244,07 & 4 & 976,30 \\
\hline \multicolumn{4}{|c|}{ AÇÃo A SER ADOTADA } \\
\hline Grau de Deterioração da Estrutura (GD) & $\mathbf{2 4 5 , 1 1}$ \\
\hline Nível de Deterioração da Estrutura & CRíTICO \\
\hline \multicolumn{4}{|c|}{} \\
\hline Prazo Máximo para Intervenção & Imediato \\
\hline \multicolumn{4}{|c|}{ IMEDIATA. } \\
\hline Inspeção especial emergencial. Planejar intervenção \\
\hline
\end{tabular}

\subsection{Discussão}

Foi observada predominância de fissuras e desplacamentos do concreto devido ao processo corrosivo nos elementos estruturais, sendo identificadas também falhas de concretagem, deformações excessivas e manchas devido à infiltração e presença de umidade de forma pontual em alguns elementos.

Todos os elementos estruturais apresentaram Gde maior que 120, com exceção de algumas lajes, que obtiveram Gde $=95$. Conforme o caderno de inspeções da metodologia GDE/UnB, tais elementos são todos caracterizados com nível de deterioração crítica (Gde > 80) e tal cenário, já esperado de acordo com o visualmente observado na inspeção, atesta a necessidade de que sejam realizadas inspeções especializadas de forma emergencial com intervenção imediata na estrutura, com seu devido escoramento e avaliação das condições de segurança de uso.

As famílias estruturais apresentaram grau de deterioração semelhante e elevado, caracterizando uma situação alarmante do ponto de vista da segurança e estabilidade estrutural. Os valores elevados contribuíram para o grau de dano associado à estrutura como um todo $(\mathrm{Gd}=245,11)$, muito maior do que o limite que caracteriza o grau crítico de deterioração $(\mathrm{Gd}=100)$. Assim, temse como classificação um grau crítico do nível de deterioração da estrutura, que incita a necessidade de intervenções imediatas pois o estado avançado de degradação já compromete a usabilidade da edificação e representa sérios riscos para a segurança dos usuários.

\section{CONCLUSÕES}

Conforme o exposto, a estrutura vistoriada apresentou sérios sinais de degradação do concreto armado, associados principalmente à ocorrência de corrosão das armaduras em estágio avançado, com desplacamento do concreto e sinais de deformação excessiva dos elementos estruturais, que podem estar associados à perda de capacidade resistente dos elementos. Tais ocorrências podem ter sido potencializadas pela ação combinada dos fenômenos deletérios como a carbonatação do concreto e ingresso de íons cloreto devido às características inadequadas da estrutura (cobrimento deficiente) frente ao seu uso e ambiente altamente agressivo onde está inserida.

Com auxílio da metodologia GDE/UnB foi possível avaliar isoladamente cada elemento estrutural afetado e a estrutura como um todo, sendo constatada criticidade na situação da estrutura frente ao uso a que se destina.

Todos os elementos avaliados foram identificados com estágio sofrível ou crítico de deterioração conforme as tabelas propostas pelo caderno de inspeção da metodologia GDE/UnB e, desta forma, a estrutura, como um todo, também foi classificada em estado crítico. Tal resultado aponta para 
uma necessidade de intervenção recuperativa imediata, devendo ser realizadas análises aprofundadas quanto ao estado de degradação do concreto e das armaduras (ex. ensaios de carbonatação e análise de perda de seção resistente do aço) para que sejam determinados os procedimentos de recuperação mais adequados ao caso e, se necessário, execução de reforços estruturais.

Observou-se que a metodologia GDE/UnB fornece indicativos valiosos que podem auxiliar no planejamento e execução da manutenção, recuperação e reabilitação das estruturas ao avaliar não apenas o grau geral de deterioração, mas também as famílias estruturais e elementos individuais. Desta forma, torna-se possível a priorização da intervenção naqueles elementos mais relevantes para a segurança estrutural em situações críticas e planejamento de futuras intervenções e manutenções em outros elementos que apresentem nível de deterioração sofrível, ou inferior, mas que ainda não comprometem a segurança e utilização da estrutura.

Além disso, os indicadores de deterioração também possibilitam que seja feito o acompanhamento do estado das estruturas de forma preventiva por meio de inspeções regulares, permitindo que fenômenos deletérios atuantes sejam identificados e controlados antes que comprometam o desempenho esperado das edificações.

\section{AGRADECIMENTOS}

Agradecemos à Coordenação de Aperfeiçoamento de Pessoal de Nível Superior (CAPES) pelo apoio e incentivo à pesquisa.

\section{REFERENCIAS}

ABNT - Associação Brasileira de Normas Técnicas. (2014). NBR 6118: Projeto de estruturas de concreto - Procedimento. Rio de Janeiro.

ABNT - Associação Brasileira de Normas Técnicas. (2013). NBR 15575-1: Edificações habitacionais: desempenho - Requisitos gerais. Rio de Janeiro.

Castro, E. K. (1994). "Desenvolvimento de metodologia para manutenção de estruturas de concreto armado". Dissertação (Mestrado) - Departamento de Engenharia Civil, Universidade de Brasília, Brasília, DF.

Fonseca, R. P. (2007). "A estrutura do instituto central de ciências: aspectos históricos, científicos e tecnológicos de projeto, execução, intervenções e proposta de manutenção". Dissertação (Mestrado em Estruturas e Construção Civil) - Universidade de Brasília, Brasília.

Galvão, R. R., Silva, F. G. S. (2021). “Avaliação de Degradação de Estruturas de Concreto em Duas Garagens de Edificios Residenciais pelo Método GDE/UnB" in: XVII Congresso Internacional sobre Patologia e Reabilitação das Construções (CINPAR), Fortaleza (Brasil), pp. 699-706.

Gasparetto, A., Pantoja, J. C., R., F. B. (2021). "Metodologia para inspeção e avaliação da segurança e durabilidade de estruturas de concreto armado". Brazilian Journal of Development, v. 7, n. 1, p. 4942-4960. 
Lima, H. J. N., Ribeiro, R. da S., Melo, G. S. S. de A., \& Palhares, R. de A. (2019). "Analysis of pathological manifestations of concrete in urban overpasses". Revista ALCONPAT, 9(2), 247 - 259. https://doi.org/10.21041/ra.v9i2.308

Oliveira, F. D., Moreira, L. B., Damaceno, J. R., Teixeira, T. D. S., Silva, J. R. (2018). “A estrutura da UEG (CCET): projeto, execução, intervenções e proposta de estratégias para manutenção (prédios adjacentes)". Revista Mirante, v. 11, n. 8.

Possan, E., Demoliner, C. A. (2013). “Desempenho, durabilidade e vida útil das edificações: abordagem geral". Revista técnico-científica, v. 1, n. 1 .

Villanueva, M. M. (2015). “A importância da manutenção preventiva para o bom desempenho da edificação". Trabalho de Conclusão de Curso. Universidade Federal do Rio de Janeiro. Rio de Janeiro. 